\title{
Darstellung von Didehydropyridinen aus (Trimethylsilyl)pyridinen
}

\author{
Franz Effenberger* und Wolfgang Daub ${ }^{1)}$ \\ Institut für Organische Chemie der Universität Stuttgart, \\ Pfaffenwaldring 55, W-7000 Stuttgart 80
}

Eingegangen am 27. März 1991

Key Words: Didehydropyridines, synthesis of

\section{Preparation of Didehydropyridines from (Trimethylsilyl)pyridines}

Halogen-substituted (trimethylsilyl)pyridines 2, 3, 5-7 and trifluoromethylsulfonyloxy-substituted (trimethylsilyl)pyridines $9 b, 11 b$ are obtained from 2- and 3-halopyridines 1, 4 or hydroxypyridines $\mathbf{8}, \mathbf{1 0}$, and 12. Reactions of the 3- and 2-(trimethylsilyl)pyridines $2,9 \mathrm{~b}$ and $11 \mathrm{~b}$ with bases in the presence of furans 15 give only protodesilylation or hydrolysis products but no indication is found for the formation of a 2,3-didehy- dropyridine. 3-Bromo-4-(trimethylsilyl)pyridine (5a) reacts with $\mathrm{KOCMe}_{3}$ in the presence of furan (15a) to give a mixture of products from which the isoquinoline derivative 20 and the tert-butoxypyridines 23,24 are formed by addition to 3,4-didehydropyridine. Under comparable conditions far higher yields of 3,4-didehydropyridines are obtained by treatment of the 3-halo-2,4-bis(trimethylsilyl)pyridines 7 with strong bases.
Didehydroheteroaromaten (Hetarine) wurden als mögliche Reaktionszwischenstufen vielfach diskutiert ${ }^{2}$. Das als erstes postulierte und am besten gesicherte Hetarin ist 3,4-Didehydropyridin, das aus verschiedenen Vorstufen zugänglich ist und mit Dienen abgefangen werden konnte ${ }^{2 \mathrm{c}, 3)}$; der erste direkte Nachweis gelang IR-spektroskopisch bei der Photolyse von Pyridin-3,4-dicarbonsäureanhydrid in einer Matrix bei $13 \mathrm{~K}^{4}$ ). In einer neueren Arbeit wurde über die Addition von Esterenolaten an 3,4-Didehydropyridin unter Bildung von Cyclobutanen berichtet ${ }^{5)}$. Für 2,3-Didehydropyridin gibt es bisher keine experimentellen Beweise ${ }^{6)}$; aus Berechnungen geht hervor, $\mathrm{da} B$ es gegenüber dem 3,4-Derivat deutlich benachteiligt sein sollte ${ }^{2 b, 7)}$.

In der vorstehenden Arbeit ${ }^{8)}$ haben wir über die Darstellung von Dehydrobenzolen aus geeignet substituierten Aryltrimethylsilanen mit Basen berichtet. Die relativ milden Reaktionsbedingungen bei dieser Eliminierungsmethode sollten auch für die Darstellung von Didehydroheteroaromaten von Vorteil sein, so daß z. B. Isomerisierungen oder Ringöffnungs-Reaktionen vermieden werden könnten. Wir beschreiben in der vorliegenden Arbeit eine neue Synthese für 3,4-Didehydropyridin und Versuche zur Darstellung des 2,3-Didehydropyridins.

Darstellung von Halogen- und (Trifluormethylsulfonyloxy)(trimethylsilyl)pyridinen

ortho-Halogen(trimethylsilyl)pyridine sind über eine selektive Metallierung geeignet substituierter Pyridine und anschließende Umsetzung mit Trimethylsilylchlorid (TMSC)

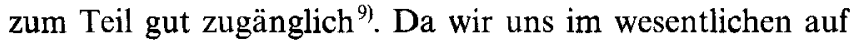
die Darstellung von 2,3- und 3,4-Didehydropyridin beschränken wollten, haben wir zunächst 2-Halogen(trimethylsilyl)- und 3-Halogen(trimethylsilyl)pyridine ausgehend von den entsprechenden Halogenpyridinen durch Metallierung und Silylierung der gebildeten Pyridyl-Anionen dargestellt ${ }^{\text {9), }}$, wobei den Reaktionsbedingungen entscheidende Bedeutung für die angestrebten Selektivitäten zukommt
(Schema 1). Tab. 1 enthält die für eine größtmögliche Selektivität optimierten Bedingungen.

Schema 1
1

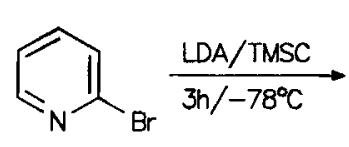<smiles>CSc1cccnc1Br</smiles>

2<smiles>CS(=O)(=O)c1ccnc(Br)c1</smiles>

3<smiles>C[Si](C)(C)c1ccncc1Br</smiles>

Bei der Darstellung von 2-Brom-3-(trimethylsilyl)pyridin (2) aus 2-Brompyridin (1) und Lithiumdiisopropylamid (LDA) nach Lit. ${ }^{9 b)}$ wurde ein Produktgemisch aus 2 (Hauptprodukt) und 2-Brom-4-(trimethylsilyl)pyridin (3) (Nebenprodukt) erhalten, aus dem 2 durch Destillation über eine Ringspaltkolonne in 49proz. Ausbeute mit 90proz. Reinheit abgetrennt werden konnte. Das isomere 3 wurde über sein Folgeprodukt bei der Umsetzung mit Benzaldehyd als (2Brom-4-pyridinyl)phenylmethanol identifiziert (s. exp. Teil).

Da nach der beschriebenen Methode zur Darstellung von 2 das zunächst entstehende Pyridyl-Anion mit TMSC zum Silylpyridin weiterreagiert, sollte die Anionen-Stabilisierung für die Produktbildung entscheidend sein, was zu der auch 
Tab. 1. Metallierung und Silylierung von 2-Brom- (1), 3-Brom- (4a) und 3-Chlorpyridin (4b) mit Lithiumdiisopropylamid (LDA) und Trimethylsilylchlorid (TMSC) in Tetrahydrofuran (THF)/Hexan

\begin{tabular}{ccccc}
\hline $\begin{array}{c}\text { Ausgangs- } \\
\text { verbin- } \\
\text { dung }\end{array}$ & $\begin{array}{c}\text { Reaktions- } \\
\text { zeit } \\
{[\mathrm{h}]}\end{array}$ & $\begin{array}{c}\text { temp. } \\
{\left[{ }^{\circ} \mathrm{C}\right]}\end{array}$ & $\begin{array}{c}\text { Produkte } \\
\text {-(trimethylsilyl)pyridin }\end{array}$ & $\begin{array}{c}\text { Ausb. } \\
(\%)\end{array}$ \\
\hline $\mathbf{1}^{\text {b) }}$ & 3 & -78 & 2-Brom-3- (2) & \\
$\mathbf{4 a}^{\mathrm{d})}$ & $1 \frac{1}{2}$ & -100 & 3-Brom-4- (5a) & 49 \\
$\mathbf{4 a}^{\text {e) }}$ & $1 \frac{1}{2}$ & -65 & 3-Brom-2,4-bis- (7a) & 68 \\
$\mathbf{4 b}^{\text {e) }}$ & $1 \frac{1}{2}$ & -65 & 3-Chlor-2,4-bis- (7b) & 55 \\
\hline
\end{tabular}

a) Anschließend ließ man auf Raumtemp. erwärmen $(16 \mathrm{~h})$. b) Zutropfen von 1/TMSC/THF zu LDA/THF/Hexan. - ${ }^{\text {c) Enthielt }}$ noch $10 \%$ 2-Brom-4-(trimethylsilyl)pyridin (3). - d) Zutropfen von 4a, dann von TMSC zu LDA/THF/Hexan. - e) Zutropfen von TMSC/THF, dann von $4 a / T H F$ zu LDA/THF/Hexan.

experimentell gefundenen bevorzugten Bildung von 2 gegenüber 3 führt. Versuche, die Umsetzung von 1 zu 2 nach der „In-situ“-Methode ${ }^{10)}$ (Metallierung in Gegenwart von TMSC) durchzuführen, um dadurch den Produkt-Anteil an 2 gegenüber 3 noch weiter zu erhöhen, waren nicht erfolgreich, obwohl dies aufgrund neuerer Rechnungen ${ }^{17)} \mathrm{zu}$ erwarten gewesen wäre.

Bei der Darstellung von 3-Brom-4-(trimethylsilyl)pyridin (5a) aus 3-Brompyridin (4a) wurde bei noch tieferen Temperaturen $\left(-100^{\circ} \mathrm{C}\right)$ gearbeitet, um mögliche Nebenreaktionen - wie Eliminierung oder Halogenwanderung ${ }^{\text {aa) }}$ - zu vermeiden. Das auf diese Weise erhaltene 5a enthielt geringe Beimengungen, vermutlich an isomerem 6a; die Isolierung von 5a mit 99proz. Reinheit (nach Kap.-GC) gelang wiederum mittels Spaltrohrdestillation in 68proz. Ausbeute. Wesentlich höhere Anteile an 6a entstehen bei der "In-situ“Methode. Die Darstellung der 2,4-disilylierten Halogenpyridine $\mathbf{7 a}, \mathbf{b}$ aus den 3-Halogenpyridinen $\mathbf{4 a}, \mathbf{b}$ erfolgte bei $-65^{\circ} \mathrm{C}$ und bereitete keine Schwierigkeiten.

Als sehr bedeutend hatte sich der Einfluß der Austrittsgruppe bei der Arin-Gewinnung aus Halogen(trimethylsilyl)benzolen erwiesen ${ }^{8)}$. Wir haben deshalb die bisher in der Literatur noch nicht beschriebenen (Trifluormethylsulfonyloxy)(trimethylsilyl)pyridine $\mathbf{9 b}$ und $\mathbf{1 1 b}$, die aufgrund der exzellenten Austrittstendenz des Trifluormethylsulfonats ${ }^{12}$ für Eliminierungsreaktionen besonders gut geeignet sein sollten, nach der für die vergleichbaren Benzol-Derivate angewandten Methode ${ }^{13)}$ über die entsprechenden Trimethylsiloxy-Derivate dargestellt.

Ausgehend von 2-Pyridon (8) bzw. 3-Hydroxypyridin (10) wurden durch Metallierung mit LDA und Umsetzung mit TMSC ausschließlich die zweifach silylierten Verbindungen 9a und 11 a erhalten, die dann über eine $O$-Metallierung mit Butyllithium mit Trifluormethansulfonsäureanhydrid zu den gewünschten Trifluormethylsulfonyloxy-substituierten (Trimethylsilyl)pyridinen $9 \mathrm{~b}$ und $11 \mathrm{~b}$ umgesetzt wurden. Die dabei resultierenden, sehr unbefriedigenden Ausbeuten (35\% 9b und $13 \%$ 11b) sind offensichtlich bedingt durch die vielfältig möglichen Folgereaktionen von $9 \mathrm{~b}$ bzw. 11b.

Bei der Silylierung von 4-Pyridon (12) nach der „In-situ“"Methode $^{10)}$ wurde das gewünschte 4-(Trimethylsiloxy)-3(trimethylsilyl)pyridin (13) als Hauptprodukt neben 4-(Tri-

Schema 2<smiles>O=c1cccc[nH]1</smiles>

8
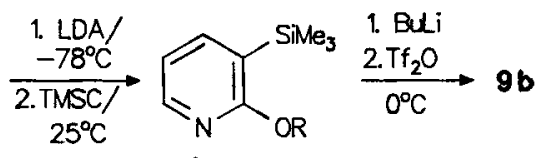

90

$$
\begin{array}{c|cc}
9.11 & \text { a } & \text { b } \\
\hline R & \mathrm{SiMe}_{3} & \mathrm{SO}_{2} \mathrm{CF}_{3}
\end{array}
$$<smiles>Oc1cccnc1</smiles>

10<smiles>O=c1cc[nH]cc1</smiles>

12

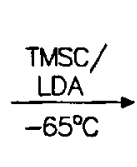

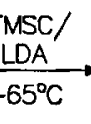

13<smiles>COc1ccncc1S(C)(C)C</smiles><smiles>[R]Oc1cccnc1S(C)(=O)=O</smiles>

1 Buli

$\underset{\mathrm{O}^{\circ} \mathrm{C}}{\stackrel{2 . \mathrm{Tf}_{2} \mathrm{O}}{\longrightarrow}} 11 \mathrm{~b}$

110

$$
\mathrm{Tf}_{2} \mathrm{O}=\left(\mathrm{CF}_{3} \mathrm{SO}_{2}\right)_{2} \mathrm{O}
$$

methylsiloxy)pyridin (14) erhalten. Alle Versuche, 13 unter den bei der Darstellung von 9b bzw. 11b angewandten Bedingungen in 4-(Trifluormethylsulfonyloxy)-3-(trimethylsilyl)pyridin zu überführen, blieben erfolglos; es entstanden nur undefinierbare Kondensationsprodukte, wie es für Pyridin-Derivate mit guten Austrittsgruppen in 4-Position bekannt ist ${ }^{14)}$.

\section{Versuche zur Darstellung von 2,3-Didehydropyridin}

Die für eine Darstellung von 2,3-Didehydropyridin geeigneten (Trimethylsilyl)pyridine 2, 9b und $11 \mathrm{~b}$ wurden mit äquimolaren Mengen Base unter den Bedingungen, die bei vergleichbaren Benzol-Derivaten zur Bildung von Dehydrobenzol geführt hatten ${ }^{8)}$, in Gegenwart der Furane 15 umgesetzt. Hierbei haben wir lediglich die Bildung der Protodesilylierungs- und Hydrolyse-Produkte 1,17 bzw. 18 beobachtet und mittels GC nachgewiesen, in keinem Fall jedoch Folgeprodukte des 2,3-Didehydropyridins in Form der mit 15 entstehenden Abfangprodukte 16.

In Gegenwart von Benzaldehyd wurde für die Umsetzung von 2 mit Tetrabutylammoniumfluorid als starker Base die $\mathrm{Si}-\mathrm{C}$-Bindungsspaltung zum 2-Brom-substituierten 3-Pyridyl-Anion $\mathbf{A}$ eindeutig nachgewiesen: dieses Anion $\mathbf{A}$ wird dabei durch Benzaldehyd unter Bildung des Carbodesilylierungsproduktes (2-Brom-3-pyridinyl)phenyl(trimethylsiloxy)methan (19a) abgefangen. Da hierbei teilweise schon eine Silyletherspaltung zu 3-(2-Brompyridyl)phenylmethanol (19b) erfolgt, haben wir in wäßrig/saurem Medium aufgearbeitet und auf diese Weise 19b in ca. 70proz. Gesamtausbeute isoliert. Die Bildung des Protodesilylierungsproduktes 18 bei der Umsetzung von 3-(Trimethylsilyl)-2-(trifluormethylsulfonyloxy)pyridin (9b) mit $\mathrm{Bu}_{4} \mathrm{NF}$ in Gegenwart von 2,5-Dimethylfuran (15b) kann über das entsprechende 2-(Trifluormethylsulfonyloxy)-substituierte 3-Pyridyl-Anion 
gedeutet werden, das durch Protonen abgefangen wird. Das bei dieser Reaktion außerdem in kleinen Mengen entstehende 3-(Trimethylsilyl)-2-pyridon (17) kann durch nucleophile Spaltung des Sulfonesters $18^{15)}$ entstehen oder durch nicht vollständig auszuschließende Spuren von Wasser, die im Sinne einer normalen nucleophilen Substitution mit dem Pyridin-Derivat $9 \mathrm{~b}$ reagieren.

Schema 3<smiles>[R]c1ccc([12F])o1</smiles>

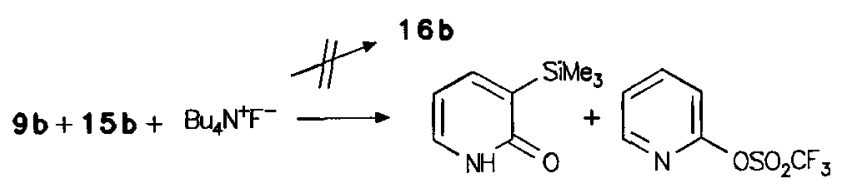
17 18<smiles></smiles>
$16 \mathrm{c}$

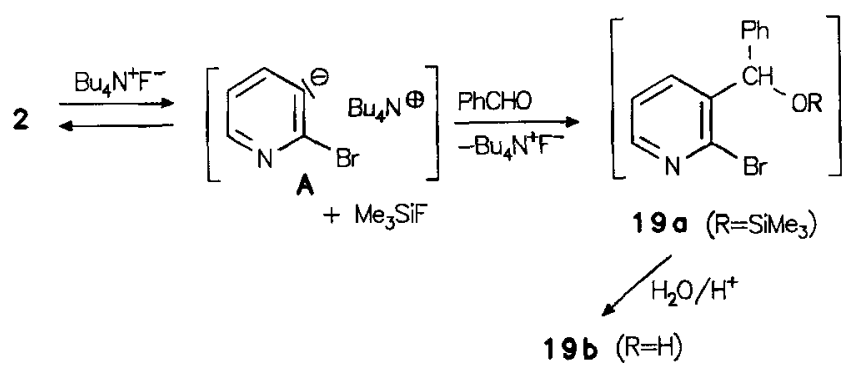

Die vorgestellten Untersuchungen zur Darstellung von 2,3-Didehydropyridin über (Trimethylsilyl)pyridine - insbesondere die Folgeprodukte der Einwirkung von Basen auf die Verbindungen $\mathbf{2}$ und $\mathbf{9 b}$ - bestätigen die größere Stabilität eines 3-Pyridyl-Anions gegenüber einem 2-PyridylAnion ${ }^{11.16)}$. So konnte auch durch Umsetzung von 3-(Trifluormethylsulfonyloxy)-2-(trimethylsilyl)pyridin (11b) mit Kalium-tert-butylat $\left(\mathrm{KOCMe}_{3}\right)$ in Gegenwart von 1,3-Diphenylisobenzofuran (15c) kein entsprechendes 2,3-Didehydro-Abfangprodukt 16c nachgewiesen werden. Die schon durch Rechnungen ${ }^{2 b, 7)}$ vorausgesagte Instabilität des 2,3Didehydropyridins wurde somit experimentell bestätigt. Ein durch Folgereaktionen eindeutig nachgewiesenes 3-Pyridyl-Anion führt - selbst wenn es nachbarständig die guten Austrittsgruppen Bromid bzw. Trifluormethylsulfonat trägt - nicht zu einer Eliminierung.

\section{Darstellung und Folgereaktionen von 3,4-Didehydropyridin}

Bei allen in der Literatur beschriebenen Untersuchungen zur Darstellung von 3,4-Didehydropyridin über eine Metallierung entsprechender Halogenpyridine sind die Ausbeuten an Abfangprodukten des Hetarins relativ niedrig, weil eine Vielzahl von Nebenprodukten gebildet wird ${ }^{3 a, 17}$. Es war deshalb nicht überraschend, daß wir bei der Umsetzung der 3-Halogen-4-(trimethylsilyl)pyridine 5 mit $\mathrm{KOCMe}_{3}$ in Gegenwart von Furan (15a) nur geringe Ausbeuten des aus dem Hetarin mit 15a gebildeten Abfangproduktes 5,8-Dihydro-5,8-epoxyisochinolin (20) erhielten (Schema 4): Mit der Brom-Verbindung 5a als Edukt wurden z. B. neben dem erwarteten 20, das in 7 proz. Ausbeute isoliert wurde, die beiden 3- und 4-Brompyridine 4a und 21 und das zu 5a isomere 4-Brom-3-(trimethylsilyl)pyridin (22) sowie die 3und 4-(tert-Butoxy)pyridine (23 und 24) mittels GC/MSAnalyse nachgewiesen.

Schema 4<smiles>COc1ccncc1OCC[18O]c1cccnc1</smiles>

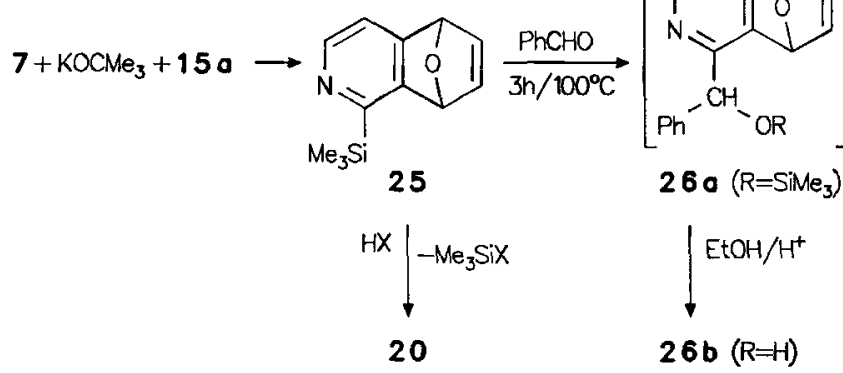

Dieses Ergebnis deckt sich mit den angeführten Literaturbefunden ${ }^{3 a, 17)}$ und hat seinen Grund wohl darin, da $B$ die Pyridyl-Anionen stabil genug sind, um auch bei relativ hohen Temperaturen nicht nur spontan Halogenid zum Dehydropyridin abzuspalten, sondern auch andere Folgereaktionen einzugehen. Damit können auch die bei der Umsetzung von 5a mit $\mathrm{KOCMe}_{3}$ in Gegenwart von 15a beobachteten Isomerisierungen des Anions zu 22 und die Bildung der Protodesilylierungsprodukte 4a und 21 gedeutet werden; das Entstehen der Substitutionsprodukte 23 und 24 wird über eine zur Addition von 15a konkurrierende Addition von tert-Butylat an 3,4-Didehydropyridin leicht verständlich.

Unerwartet verliefen die Umsetzungen der 3-Halogen-2,4bis(trimethylsilyl)-Verbindungen 7 mit $\mathrm{KOCMe}_{3}$ in Gegen- 
wart von 15a: in vergleichsweise guter Ausbeute (42\%) konnten wir aus der Bromverbindung 7a das 5,8-Dihydro1-(trimethylsilyl)-5,8-epoxyisochinolin (25) als Abfangprodukt des 3,4-Didehydropyridins bei quantitativem Umsatz in reiner Form isolieren, als einziges Nebenprodukt entstand noch dessen Protodesilylierungsprodukt 20, das im Rohprodukt mit ca. $10 \%$ bezogen auf 25 mittels GC nachgewiesen wurde. Daß die Trimethylsilyl-Gruppe in 25 nicht nur durch Protonen, sondern auch durch andere Elektrophile substituiert werden kann, zeigt die mit Benzaldehyd bei höherer Temperatur $\left(100^{\circ} \mathrm{C}\right)$ erfolgende Carbodesilylierung von $25 \mathrm{zu}$ 5,8-Dihydro-1-[ $\alpha$-(trimethylsiloxy)benzyl]5,8-epoxyisochinolin (26a), das bei der Aufarbeitung unter Silyletherspaltung zu (5,8-Dihydro-5,8-epoxyisochinolin-1yl)phenylmethanol (26b) führt. Die Struktur von 26b konnte NMR-spektroskopisch eindeutig gesichert werden. Überraschenderweise lagern die Verbindungen 26 unter den angewandten Bedingungen (EtOH/HCl unter Rückfluß) nicht in die entsprechenden Hydroxychinoline um. Als Ursache für die mit deutlich besseren Ausbeuten verlaufende Didehydropyridin-Bildung ausgehend von den in 2- und 4-Stellung Trimethylsilyl-substituierten Pyridinen 7 im Vergleich zu den nur in 4-Stellung Trimethylsilyl-substituierten Pyridinen 5 nehmen wir eine sterische Unterstützung der Halogenid-Eliminierung in 3-Stellung durch die TrimethylsilylGruppe in 2-Position des Pyridins an.

Die einfach zugänglichen 3-Halogen-2,4-bis(trimethylsilyl)pyridine 7 stellen somit als Ausgangsprodukte für die Darstellung von 3,4-Didehydropyridinen eine interessante Alternative zu den bisher bekannten Synthesen dar; von Vorteil erweist sich dabei die im Molekül vorhandene Trimethylsilyl-Gruppe, die noch vielfältige Folgereaktionen ${ }^{16)}$ eingehen kann.

Wir danken Herrn $T$. Beck für die Durchführung vorbereitender Versuche zur Darstellung von Didehydropyridinen, der Deutschen Forschungsgemeinschaft und dem Fonds der Chemischen Industrie für die Unterstützung dieser Arbeit.

\section{Experimenteller Teil}

'H-NMR: Varian EM 360 und T 60 sowie Bruker WP 80 und CXP 300, TMS als interner Standard. - GC: a) Hewlett-Packard $5700 \mathrm{~A}$ und $5710 \mathrm{~A}$ mit FID, Trägergas: Reinstickstoff: Glassäulen $2.3 \mathrm{~m} \times 2 \mathrm{~mm}$, Phasen: OV 17, 101 und CW $20 \mathrm{M}$ auf Chromosorb W. b) Carlo-Erba-Fractovap GI 4160 mit FID, Trägergas 0.7 bar Helium; Glaskapillaren $20 \mathrm{~m}$, Phasen SE-52 und SE-54. - Präparative Säulenchromatographie: Glassäule $20 \mathrm{~cm} \times 3 \mathrm{~cm}$ mit Kieselgel 60, 230-400 mesh ASTM (Macherey-Nagel). - EI-MS: Varian MAT 711. - GC/MS: a) Carlo-Erba, Fractovap 2151, ACSpezial der Firma Brechbühler AG, Urdorf; b) Finnigan 4023 mit Incos-Datensystem 2300, Trennkapillare: $20 \mathrm{~m}$ belegt mit SE-52 und SE-54.

\section{Metallierung und Silylierung von Halogenpyridinen}

1) Von 2-Brompyridin (1) nach Lit. ${ }^{9 b)}$ : Man tropft bei $-78^{\circ} \mathrm{C}$ unter Rühren zu $0.25 \mathrm{~mol}$ Lithiumdiisopropylamid (LDA), dargestellt aus $25.3 \mathrm{~g}$ Diisopropylamin in $100 \mathrm{ml}$ Tetrahydrofuran (THF) und $160 \mathrm{ml}$ einer $1.6 \mathrm{~m}$ Lösung von Butyllithium in Hexan, innerhalb von $3 \mathrm{~h}$ die Mischung aus $39.5 \mathrm{~g}(0.25 \mathrm{~mol}) 1$ und $26.8 \mathrm{~g}(0.25$ mol) Trimethylsilylchlorid (TMSC) in $50 \mathrm{ml}$ THF, läßt unter Rüh- ren auf Raumtemp. erwärmen (16 h), gießt das Gemisch in $100 \mathrm{ml}$ einer ges. Ammoniumchlorid-Lösung, trennt die organische Phase ab und extrahiert die wäßrige Phase dreimal mit jeweils $100 \mathrm{ml}$ Diethylether. Nach Trocknen der vereinigten organischen Phasen mit Magnesiumsulfat und Einengen wird der Rückstand über eine Spaltrohrkolonne i. Vak. destilliert. Ausb. 28.1 g 2-Brom-3-(trimethylsilyl) pyridin (2), Sdp. $120-124^{\circ} \mathrm{C} / 15$ Torr (Lit. ${ }^{9 b)} 105^{\circ} \mathrm{C} / 3.5$ Torr), das ca. 10\% 2-Brom-4-(trimethylsilyl)pyridin (3) enthielt (mittels GC bestimmt); ${ }^{1} \mathrm{H}-\mathrm{NMR}$-spektroskopisch identisch mit 2 in Lit. ${ }^{9 b)}$, die Signale von 3 waren gerade noch erkennbar.

\section{2) Von 3-Brompyridin (4a)}

a) Man tropft bei $-100^{\circ} \mathrm{C}$ unter Rühren zu $51.5 \mathrm{mmol} \mathrm{LDA}$, hergestellt aus $5.21 \mathrm{~g}$ Diisopropylamin in $100 \mathrm{ml}$ THF und $32.5 \mathrm{ml}$ einer $1 \mathrm{M}$ Lösung von Butyllithium in Hexan, innerhalb von $30 \mathrm{~min}$ $7.9 \mathrm{~g}(50.0 \mathrm{mmol}) 4 \mathrm{a}$, rührt $1 \mathrm{~h}$, tropft dann $10.9 \mathrm{~g}(100 \mathrm{mmol})$ TMSC zu und läßt unter Rühren auf Raumtemp. erwärmen (16 h). Nach Zugabe von $100 \mathrm{ml}$ Hexan wird der Niederschlag abfiltriert. Das Reaktionsgemisch - es enthielt neben 3-Brom-4-(trimethylsilyl)pyridin (5a) ein Isomeres [vermutlich 3-Brom-2-(trimethylsi1yl)pyridin (6a)] im Verhältnis 10:1 (mittels Kap.-GC bestimmt) wird im Rotationsverdampfer eingeengt und der Rückstand i. Vak. über eine Spaltrohrkolonne destilliert, Ausb. $7.83 \mathrm{~g}$ 5a, Sdp. $117-120^{\circ} \mathrm{C} / 11$ Torr. $-{ }^{1} \mathrm{H}-\mathrm{NMR}\left(\mathrm{CDCl}_{3}\right): \delta=8.60(\mathrm{~s}, 1 \mathrm{H}, 1-\mathrm{H})$, $8.41\left(\mathrm{~d}, J_{5,6}=4.4 \mathrm{~Hz}, 1 \mathrm{H}, 6-\mathrm{H}\right), 7.26(\mathrm{~d}, 1 \mathrm{H}, 5-\mathrm{H}), 0.40[\mathrm{~s}, 9 \mathrm{H}$, $\left.\mathrm{Si}\left(\mathrm{CH}_{3}\right)_{3}\right]$.

$$
\begin{array}{lll}
\mathrm{C}_{8} \mathrm{H}_{12} \mathrm{BrSi} \text { (230.2) } & \text { Ber. C } 41.74 \text { H } 5.25 \mathrm{Br} 34.71 \text { N } 6.08 \\
& \text { Gef. C } 41.85 \text { H } 5.27 \mathrm{Br} 34.26 \text { N } 6.19
\end{array}
$$

b) Man tropft $\mathrm{zu} 10.9 \mathrm{~g}(100.0 \mathrm{mmol})$ TMSC und $7.90 \mathrm{~g} \mathrm{(50.0}$ mmol) $4 \mathrm{a}$ in $50 \mathrm{ml} \mathrm{THF}$ bei $-65^{\circ} \mathrm{C}$ (Innentemp.) unter Rühren innerhalb von $30 \mathrm{~min} 51.5 \mathrm{mmol}$ LDA, hergestellt aus $5.21 \mathrm{~g}$ Diisopropylamin und $32.5 \mathrm{ml}$ einer $1.6 \mathrm{M}$ Lösung von Butyllithium in Hexan, und läßt das Reaktionsgemisch unter Rühren auf Raumtemp. erwärmen (16 h). Nach Zugabe von $100 \mathrm{ml}$ Hexan wird wie unter a) beschrieben aufgearbeitet, die Vakuumdestillation über eine Spaltrohrkolonne ergab 4 Fraktionen. 1. Fraktion: $0.87 \mathrm{~g}$ $(10 \%) 5 a$, das ca. $13 \%$ 6a enthielt (mittels GC bestimmt), Sdp. bis $74^{\circ} \mathrm{C} / 12$ Torr. - 2. Fraktion: Ausb. 2.5 g (22\%) 6a, das ca. $20 \%$ 5a enthielt (mittels GC bestimmt), Sdp. $74-112^{\circ} \mathrm{C} / 12$ Torr. $-{ }^{1} \mathrm{H}$ NMR $\left(\mathrm{CDCl}_{3}\right): \delta=8.70\left(\mathrm{dd}, J_{5,6}=4.8, J_{4,6}=1.6 \mathrm{~Hz}, 1 \mathrm{H}, 6-\mathrm{H}\right)$, $7.70\left(\mathrm{dd}, J_{4,5}=8.0 \mathrm{~Hz}, 1 \mathrm{H}, 4-\mathrm{H}\right), 7.00\left(\mathrm{dd}, J_{4,5}=8.2 \mathrm{~Hz}, 1 \mathrm{H}\right.$, 5-H), 0.24 [s, 9H, Si( $\left.\left.\mathrm{CH}_{3}\right)_{3}\right]$. - 3. Fraktion: Ausb. $4.79 \mathrm{~g}(42 \%) 5 \mathrm{a}$ (Reinheit $92 \%$, mittels GC bestimmt), Sdp. $112-124^{\circ} \mathrm{C} / 12$ Torr; ${ }^{1}$ H-NMR-spektroskopisch identisch mit dem unter a) beschriebe-

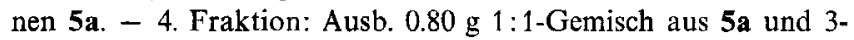
Brom-2,4-bis(trimethylsilyl)pyridin (7a) (mittels GC bestimmt), Sdp. $125-148^{\circ} \mathrm{C} / 12$ Torr.

\section{3-Halogen-2,4-bis (trimethylsilyl)pyridine 7}

Allgemeines: Man tropft zu der Lösung von LDA bei $-65^{\circ} \mathrm{C}$ (Badtemp.) unter Rühren innerhalb von $30 \mathrm{~min}$ die Lösung von TMSC in THF und dann dic des 4-Halogenpyridins 4 in THF, rührt $1 \mathrm{~h}$, tropft $1 \mathrm{ml}$ Wasser zu, läßt das Gemisch auf Raumtemp. erwärmen (16 h), gießt auf Eis, trennt die organische Phase ab und extrahiert die wäßrige Phase dreimal mit jeweils $100 \mathrm{ml}$ Diethylether. Nach Trocknen der vereinigten Extrakte mit Natriumsulfat und Einengen wird der Rückstand i. Vak. über eine Spaltrohrkolonne destilliert.

3-Brom-2,4-bis (trimethylsilyl)pyridin (7a): Aus $192 \mathrm{mmol}$ LDA, hergestellt aus $19.4 \mathrm{~g}$ Diisopropylamin in $25 \mathrm{ml}$ THF und $120 \mathrm{ml}$ einer 1.6 M Lösung von Butyllithium in Hexan, und $20.7 \mathrm{~g}$ (191 mmol) TMSC sowic $14.6 \mathrm{~g}$ (92.4 mmol) $4 \mathrm{a}$ in $75 \mathrm{ml}$ THF, Ausb. 14.5 g, Sdp. $105-113^{\circ} \mathrm{C} / 0.1$ Torr, Schmp. $51-52^{\circ} \mathrm{C}$ (Pentan). - 
${ }^{1} \mathrm{H}-\mathrm{NMR}\left(\mathrm{CDCl}_{3}\right): \delta=8.65\left(\mathrm{~d}, J_{5,6}=4.8 \mathrm{~Hz}, 1 \mathrm{H}, 6-\mathrm{H}\right), 7.20(\mathrm{~d}$, $1 \mathrm{H}, 5-\mathrm{H}), 0.46$ und $0.41\left[2 \mathrm{~s}, 18 \mathrm{H}, 2 \mathrm{Si}\left(\mathrm{CH}_{3}\right)_{3}\right]$.

$\mathrm{C}_{11} \mathrm{H}_{20} \mathrm{BrNSi}_{2}$ (302.4) Ber. C $43.69 \mathrm{H} 6.67 \mathrm{Br} 26.43 \mathrm{~N} 4.63$ Gef. C 43.78 H 6.75 Br 26.14 N 4.53

3-Chlor-2,4-bis (trimethylsilyl)pyridin (7b): Aus $220 \mathrm{mmol}$ LDA, hergestellt aus $22.3 \mathrm{~g}$ Diisopropylamin und $140 \mathrm{ml}$ einer $1.6 \mathrm{M} \mathrm{Bu}$ tyllithium-Lösung in Hexan, und $23.9 \mathrm{~g} \mathrm{(220} \mathrm{mmol})$ TMSC in $50 \mathrm{ml}$ THF sowie $22.7 \mathrm{~g}$ (200 mmol) 3-Chlorpyridin (4b), Ausb. $14.6 \mathrm{~g}$ (55\%), Sdp. $107-110^{\circ} \mathrm{C} / 10$ Torr, Schmp. $48^{\circ} \mathrm{C}$ (Hexan). $-{ }^{1} \mathrm{H}-$ NMR $\left(\mathrm{CDCl}_{3}\right): \delta=8.584\left(\mathrm{~d}, J_{5,6}=4.57 \mathrm{~Hz}, 1 \mathrm{H}, 6-\mathrm{H}\right), 7.243(\mathrm{~d}$, $\left.J_{5,6}=4.67 \mathrm{~Hz}, 1 \mathrm{H}, 5-\mathrm{H}\right), 0.415$ und $0.382\left[2 \mathrm{~s}, 18 \mathrm{H}, 2 \mathrm{Si}\left(\mathrm{CH}_{3}\right)_{3}\right]$.

$\mathrm{C}_{11} \mathrm{H}_{20} \mathrm{ClNSi}_{2}$ (257.9) Ber. C $51.23 \mathrm{H} 7.81 \mathrm{Cl} 13.75 \mathrm{~N} 5.43$ Gef. C $51.29 \mathrm{H} 7.67 \mathrm{Cl} 13.63$ N 5.38

\section{(Trifluormethylsulfonyloxy) (trimethylsilyl) pyridine}

2-(Trimethylsiloxy)-3-(trimethylsilyl) pyridin (9a): Man gibt bei $-78^{\circ} \mathrm{C}$ (Badtemp.) unter Rühren zu $0.50 \mathrm{~mol}$ LDA, hergestellt aus $50.7 \mathrm{~g}$ Diisopropylamin und $320 \mathrm{ml}$ einer $1.6 \mathrm{M}$ Lösung von Butyllithium in Hexan, portionsweise $22.1 \mathrm{~g}(0.23 \mathrm{~mol})$ 2-Pyridon $(8)$, läßt das Gemisch unter Rühren auf Raumtemp. erwärmen $(16 \mathrm{~h})$, tropft $54.5 \mathrm{~g}(0.50 \mathrm{~mol})$ TMSC zu, rührt $16 \mathrm{~h}$ und saugt den Niederschlag ab. Aus dem Filtrat wird unter Feuchtigkeitsausschluß das Lösungsmittel bei 16 Torr entfernt, der Rückstand wird i. Vak.

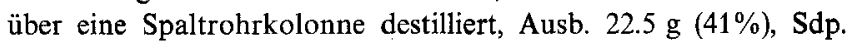
$123-137^{\circ} \mathrm{C} / 15$ Torr. $-{ }^{1} \mathrm{H}-\mathrm{NMR}\left(\mathrm{CDCl}_{3}\right): \delta=8.14$ (dd, $J_{5,6}=5$, $\left.J_{4,6}=2 \mathrm{~Hz}, 1 \mathrm{H}, 6-\mathrm{H}\right), 7.68\left(\mathrm{dd}, J_{4,5}=8 \mathrm{~Hz}, 1 \mathrm{H}, 4-\mathrm{H}\right), 6.82(\mathrm{dd}$, $1 \mathrm{H}, 5-\mathrm{H}), 0.33$ und $0.23\left[2 \mathrm{~s}, 18 \mathrm{H}, \mathrm{Si}\left(\mathrm{CH}_{3}\right)_{3}\right]$.

$$
\begin{array}{lll}
\mathrm{C}_{11} \mathrm{H}_{21} \mathrm{NOSi}_{2} \text { (239.5) } & \text { Ber. C 55.17 H 8.84 N } 5.85 \\
& \text { Gef. C 55.32 H 8.92 N } 5.64
\end{array}
$$

9a hydrolysiert an der Luft langsam zu 3-(Trimethylsilyl)-2-pyridon (17) (s. nachstehend), Schmp. $110-111^{\circ} \mathrm{C}$ (Acetonitril) (Lit. ${ }^{18)}$ $101-102^{\circ} \mathrm{C}$ ), 'H-NMR-spektroskopisch identisch mit 17 in Lit. ${ }^{18)}$.

2-(Trifluormethylsulfonyloxy)-3-(trimethylsilyl)pyridin (9b): Man tropft bei $0^{\circ} \mathrm{C} \mathrm{zu} 12.2 \mathrm{~g}(50.9 \mathrm{mmol}) 9 \mathrm{a}$ in $100 \mathrm{ml}$ Diethylether innerhalb von $10 \mathrm{~min}$ unter Rühren $35 \mathrm{ml}(56.0 \mathrm{mmol})$ einer $1.6 \mathrm{M}$ Lösung von Butyllithium in Hexan, rührt $2 \mathrm{~h}$ bei $0^{\circ} \mathrm{C}$, tropft innerhalb von $15 \mathrm{~min}$ bei $0^{\circ} \mathrm{C}$ unter Rühren $25.1 \mathrm{~g}(89.0 \mathrm{mmol})$ Trifluormethansulfonsäureanhydrid $\left(\mathrm{Tf}_{2} \mathrm{O}\right) \mathrm{zu}$, rührt $2 \mathrm{~h}$ bei $0^{\circ} \mathrm{C}$ und läßt unter Rühren auf Raumtemp. erwärmen $(16 \mathrm{~h})$. Nach Entfernen des Lösungsmittels unter Feuchtigkeitsausschluß im Rotationsverdampfer wird der Rückstand mit $100 \mathrm{ml}$ Pentan behandelt. Es wird über eine Vakuumfritte abgesaugt und das Filtrat wie im Falle von

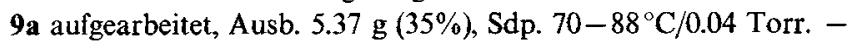
${ }^{1} \mathrm{H}-\mathrm{NMR}\left(\mathrm{CDCl}_{3}\right): \delta=8.36\left(\mathrm{dd}, J_{4,6}=4.4, J_{5,6}=2 \mathrm{~Hz}, 1 \mathrm{H}, 6-\mathrm{H}\right)$, $8.07\left(\mathrm{dd}, J_{4,5}=7.5 \mathrm{~Hz}, 1 \mathrm{H}, 4-\mathrm{H}\right), 7.34(\mathrm{dd}, 1 \mathrm{H}, 5-\mathrm{H}), 0.31[\mathrm{~s}, 9 \mathrm{H}$, $\left.\mathrm{Si}\left(\mathrm{CH}_{3}\right)_{3}\right]$.

$\mathrm{C}_{9} \mathrm{H}_{12} \mathrm{~F}_{3} \mathrm{NO}_{3} \mathrm{SSi}$ (299.3) Ber. C $36.11 \mathrm{H} 4.04 \mathrm{~N} 4.68 \mathrm{~S} 10.71$ Gef. C 36.92 H 4.25 N 4.77 S 10.67

\section{3-(Trimethylsiloxy)-2-(trimethylsilyl) pyridin (11a)}

a) Man gibt bei $-78^{\circ} \mathrm{C}$ (Badtemp.) unter Rühren zu $0.48 \mathrm{~mol}$ LDA, hergestellt aus $48.6 \mathrm{~g}(0.48 \mathrm{~mol})$ Diisopropylamin und $307 \mathrm{ml}$ einer $1.6 \mathrm{M}$ Lösung von Butyllithium in Hexan, $52.1 \mathrm{~g}(0.48 \mathrm{~mol})$ TMSC in $50 \mathrm{ml}$ THF und sofort danach portionsweise $19.0 \mathrm{~g}(0.20$ mol) 3-Hydroxypyridin (10) in $175 \mathrm{ml} \mathrm{THF}$, rührt $1 \mathrm{~h}$ bei $-78^{\circ} \mathrm{C}$, läßt unter Rühren auf Raumtemp. erwärmen $(16 \mathrm{~h})$, saugt den Niederschlag ab und arbeitet wie bei $9 \mathrm{a}$ auf, Ausb. $41.0 \mathrm{~g}(85 \%)$, Sdp. $116-118^{\circ} \mathrm{C} / 10$ Torr. $-{ }^{1} \mathrm{H}-\mathrm{NMR}\left(\mathrm{CDCl}_{3}\right): \delta=8.39$ (dd, $J_{5,6}=$ $\left.4.52, J_{4,6}=1.33 \mathrm{~Hz}, 1 \mathrm{H}, 6-\mathrm{H}\right), 7.07\left(\mathrm{dd}, J_{4,5}=8.20, J_{5,6}=4.54 \mathrm{~Hz}\right.$, $1 \mathrm{H}, 5-\mathrm{H}), 6.99\left(\mathrm{dd}, J_{4,5}=8.32, J_{4,6}=1.45 \mathrm{~Hz}, 1 \mathrm{H}, 4-\mathrm{H}\right), 0.372$ und 0.348 [jeweils s, $9 \mathrm{H}, \mathrm{CSi}\left(\mathrm{CH}_{3}\right)_{3}$ bzw. OSi $\left(\mathrm{CH}_{3}\right)_{3}$ ].

$\mathrm{C}_{11} \mathrm{H}_{21} \mathrm{NOSi}_{2}$ (239.5) Ber. C 55.17 H $8.84 \mathrm{~N} 5.85$ Gef. C 55.32 H 8.92 N 5.64
3-(Trifluormethylsulfonyloxy)-2-(trimethylsilyl) pyridin (11 b): Wie bei der Herstellung von 9b beschrieben werden zu $13.3 \mathrm{~g}(55.5$ mmol) 11a in $100 \mathrm{ml}$ Diethylether bei $0^{\circ} \mathrm{C}$ unter Rühren, jedoch innerhalb von $20 \mathrm{~min}, 54.3 \mathrm{ml}$ ( $54.3 \mathrm{mmol})$ einer $1 \mathrm{M}$ Lösung von Butyllithium in Hexan getropft. Es wird $4 \mathrm{~h}$ bei $0^{\circ} \mathrm{C}$ gerührt. Dann werden $24.0 \mathrm{~g}$ (85.1 mmol) $\mathrm{Tf}_{2} \mathrm{O}$ zugetropft, anschließend läßt man das Gemisch unter Rühren auf Raumtemp. erwärmen $(16 \mathrm{~h})$ und arbeitet wie bei 9 b auf, Ausb. $2.07 \mathrm{~g}(12.5 \%)$, Sdp. $62-66^{\circ} \mathrm{C} / 0.04$ Torr. $-{ }^{1} \mathrm{H}$-NMR $\left(\mathrm{CDCl}_{3}\right): \delta=8.69\left(\mathrm{dd}, J_{5,6}=4.57, J_{4,6}=1.2 \mathrm{~Hz}\right.$, $1 \mathrm{H}, 6-\mathrm{H}), 7.53\left(\mathrm{dd}, J_{4,5}=8.64 \mathrm{~Hz}, 1 \mathrm{H}, 4-\mathrm{H}\right), 7.24\left(\mathrm{dd}, J_{4,5}=8.66\right.$ $\mathrm{Hz}, 1 \mathrm{H}, 5-\mathrm{H}), 0.348\left[\mathrm{~s}, 9 \mathrm{H}, \mathrm{Si}\left(\mathrm{CH}_{3}\right)_{3}\right]$.

Umsetzung von 4-Pyridon (12) mit LDA und TMSC: Man gibt bei $-65^{\circ} \mathrm{C}$ (Innentemp.) unter Rühren zu $9.51 \mathrm{~g}(0.100 \mathrm{~mol}) 12$ und $43.5 \mathrm{~g}(0.400 \mathrm{~mol}) \mathrm{TMSC}$ in $80 \mathrm{ml}$ THF innerhalb von $30 \mathrm{~min}$ die Lösung von $210 \mathrm{mmol} \mathrm{LDA}$, hergestellt aus $21.3 \mathrm{~g}$ Diisopropylamin in $50 \mathrm{ml}$ THF und $131 \mathrm{ml}$ einer $1.6 \mathrm{M}$ Lösung von Butyllithium in Hexan, läßt unter Rühren auf Raumtemp. erwärmen (16 h), gibt $100 \mathrm{ml}$ Hexan zu, saugt den Niederschlag ab, entfernt aus dem Filtrat das Lösungsmittel im Rotationsverdampfer bei 16 Torr und fraktioniert den Rückstand i.Vak. über eine $20-\mathrm{cm}$ Vigreuxkolonne. 1. Fraktion: Ausb. $4.68 \mathrm{~g}$ (28\%) 4-(Trimethylsiloxy)pyridin (14), Sdp. $85-90^{\circ} \mathrm{C} / 12$ Torr. - ${ }^{1} \mathrm{H}-\mathrm{NMR}\left(\mathrm{CDCl}_{3}\right)$ : $\delta=8.35\left(\mathrm{dd}, J_{2,3}=5, J_{2,5}=2 \mathrm{~Hz}, 2 \mathrm{H}, 2-, 6-\mathrm{H}\right), 6.70(\mathrm{dd}, 2 \mathrm{H}, 3-$ ,5-H), $0.29\left[\mathrm{~s}, 9 \mathrm{H}, \mathrm{OSi}\left(\mathrm{CH}_{3}\right)_{3}\right]$. 2. Fraktion: Ausb. $9.78 \mathrm{~g} \mathrm{(41 \% )} \mathrm{4-}$ (Trimethylsiloxy) $-3-\left(\right.$ trimethylsilyl) pyridin (13), Sdp. $133-137^{\circ} \mathrm{C} /$ 12 Torr. $-{ }^{1} \mathrm{H}-\mathrm{NMR}\left(\mathrm{CDCl}_{3}\right): \delta=8.40(\mathrm{~s}, 1 \mathrm{H}, 6-\mathrm{H}), 8.36\left(\mathrm{~d}, J_{5,6}=\right.$ $5 \mathrm{~Hz}, 1 \mathrm{H}, 6-\mathrm{H}), 6.63(\mathrm{~d}, 1 \mathrm{H}, 5-\mathrm{H}), 0.34\left[\mathrm{~s}, 9 \mathrm{H}, \mathrm{CSi}\left(\mathrm{CH}_{3}\right)_{3}\right], 0.29[\mathrm{~s}$, $\left.9 \mathrm{H}, \mathrm{OSi}\left(\mathrm{CH}_{3}\right)_{3}\right]$.

$$
\begin{array}{ll}
\mathrm{C}_{11} \mathrm{H}_{21} \mathrm{NOSi}_{2} \text { (239.5) } & \text { Ber. C } 55.17 \text { H } 8.84 \text { N } 5.85 \\
& \text { Gef. C } 55.29 \text { H } 8.68 \text { N } 6.02
\end{array}
$$

\section{Versuche zur Darstellung von 2,3-Didehydropyridin}

Umsetzung von 2-Brom-3-(trimethylsilyl) pyridin (2) mit $\mathrm{KOCMe}_{3}$ in Gegenwart von Furan (15a): $0.141 \mathrm{~g}(1.26 \mathrm{mmol}) 2$ und $1 \mathrm{~g}$ (ca. $14 \mathrm{mmol}$ ) 15a in $5 \mathrm{ml}$ Hexamethylphosphorsäuretriamid (HMPT) werden in einen Kolben gefüllt, der mit einem Gummiseptum verschlossen wird. Bei $0^{\circ} \mathrm{C}$ tropft man $0.267 \mathrm{~g}(1.16 \mathrm{mmol}) 2$ langsam mit einer Spritze zu, wobei sofort Schwarzfärbung erfolgt. Nach $1 \mathrm{~h}$ wird das Gemisch in $20 \mathrm{ml}$ 2proz. HCl gegossen, mit $20 \mathrm{ml}$ Diethylether extrahiert und der Extrakt mit Magnesiumsulfat getrocknet. Gaschromatographisch wurde nur 2-Brompyridin (1) und kein 5,8Dihydro-5,8-epoxychinolin (16a) nachgewiesen.

Umsetzung von 2-(Trifluormethylsulfonyloxy)-3-(trimethylsilyl)pyridin (9b) mit TBAF und 2,5-Dimethylfuran (15b): $0.79 \mathrm{~g} \mathrm{(2.64}$ mmol) $9 \mathrm{~b}, 0.96 \mathrm{~g}$ (10.0 mmol) 15b in $10 \mathrm{ml}$ HMPT und $5.3 \mathrm{ml}(2.65$ mmol) einer $0.5 \mathrm{M}$ Lösung von TBAF in THF werden $4 \mathrm{~h}$ bei $80^{\circ} \mathrm{C}$ gerührt. Die gaschromatographische Untersuchung des Reaktionsgemisches zeigte vollständigen Umsatz zu 3-(Trimethylsilyl)-2-pyridon (17) (s. vorstehend) und 2-(Trifluormethylsulfonyloxy)pyridin (18). 5,8-Dihydro-5,8-dimethyl-5,8-epoxychinolin (16b) konnte nicht nachgewiesen werden.

Umsetzung von 3-(Trifluormethylsulfonyloxy)-2-(trimethylsilyl)pyridin (11 b) mit $\mathrm{KOCMe}_{3}$ und 1,3-Diphenylisobenzofuran (15c): Die Lösung von $6.75 \mathrm{mg}(2.5 \mathrm{mmol}) 15 \mathrm{c}$ in $10 \mathrm{ml}$ HMPT wird i. Vak. entgast und unter Eiskühlung und Rühren mit $0.75 \mathrm{~g}(2.5 \mathrm{mmol})$ $11 \mathrm{~b}$ versetzt. Innerhalb von $15 \mathrm{~min}$ läßt man die Lösung von $0.28 \mathrm{~g}$ (2.5 mmol) $\mathrm{KOCMe}_{3}$ in $1.8 \mathrm{ml} \mathrm{HMPT}$ zutropfen und rührt $16 \mathrm{~h}$ bei Raumtemp. Das Gemisch wird mit $50 \mathrm{ml}$ Wasser versetzt und dreimal mit jeweils $50 \mathrm{ml}$ Diethylether extrahiert. Die gaschromatographische Untersuchung der vereinigten Etherextrakte ergab keinen Hinweis auf die Bildung von 5,10-Dihydro-5,10-diphenyl5,10-epoxybenzo $[g]$ chinolin (16c). 
Umsetzung von 2-Brom-3-(trimethylsilyl) pyridin (2) mit TBAF in Gegenwart von Benzaldehyd: $\mathrm{Zu}$ jeweils $20.1 \mathrm{mmol}$ des bei der Umsetzung von 1 mit LDA und TMSC erhaltenen Produktes $2(4.63 \mathrm{~g})$ sowie Benzaldehyd $(2.13 \mathrm{~g})$ in $20 \mathrm{ml}$ THF werden $1 \mathrm{ml}(0.5 \mathrm{mmol})$ einer $0.5 \mathrm{M}$ Lösung von TBAF in THF gegeben, dann wird $16 \mathrm{~h}$ bei Raumtemp. gerührt, nach Zugabe von $5 \mathrm{ml}$ einer halbkonz. HCl-Lösung $16 \mathrm{~h}$ gerührt und dreimal mit jeweils $20 \mathrm{ml}$ Diethylether extrahiert. Die vereinigten Extrakte werden mit ges. Natriumhydrogencarbonat-Lösung neutralisiert, mit $20 \mathrm{ml}$ Wasser gewaschen und eingeengt, der Rückstand wird aus Hexan/Benzol (4:1) umkristallisiert. Nach Chromatographie der Mutterlauge über Kieselgel mit Hexan/Butanol $(85: 15)$ und Umkristallisation aus $\mathrm{He}$ xan/Butanol (99:1) Ausb. $3.65 \mathrm{~g}$ (69\%) (2-Brom-3-pyridinyl)phenylmethanol (19), Schmp. $124^{\circ} \mathrm{C}$ [Lit. ${ }^{96)} 116^{\circ} \mathrm{C}$ (EtOH)], ${ }^{1} \mathrm{H}-\mathrm{NMR}-$ spektroskopisch identisch mit dem Produkt in Lit. ${ }^{9 b)}$, sowie $0.106 \mathrm{~g}$ (2\%) (2-Brom-4-pyridinyl) phenylmethanol, Schmp. $105^{\circ} \mathrm{C} .-{ }^{1} \mathrm{H}-$ NMR $\left(\mathrm{CDCl}_{3}\right): \delta=8.216\left(\mathrm{~d}, J_{5,6}=4.77 \mathrm{~Hz}, 1 \mathrm{H}, 6-\mathrm{H}\right), 7.565(\mathrm{~d}$, $\left.J_{3,5}=1.26 \mathrm{~Hz}, 1 \mathrm{H}, 3-\mathrm{H}\right), 7.400-7.294(\mathrm{~m}, 5 \mathrm{H}, \mathrm{Ph}), 7.230(\mathrm{dd}$, $\left.J_{5,6}=5.18 \mathrm{~Hz}, 1 \mathrm{H}, 5-\mathrm{H}\right), 5.754(\mathrm{~d}, J=3.28 \mathrm{~Hz}, 1 \mathrm{H}, \mathrm{CH}), 2.873$ (d, $J=3.46 \mathrm{~Hz}, 1 \mathrm{H}, \mathrm{OH}$ ).

$$
\mathrm{C}_{12} \mathrm{H}_{10} \text { BrNO (264.1) Ber. C } 54.57 \text { H } 3.82 \text { Br } 30.25 \text { N } 5.30
$$
Gef. C 54.49 H 3.79 Br 30.00 N 5.19

\section{Darstellung und Folgereaktion von 3,4-Dihydropyridinen}

Umsetzung von 3-Brom-4-(trimethylsilyl)pyridin (5a) mit $\mathrm{KOCMe}_{3}$ und 15a

a) Man tropft bei $0^{\circ} \mathrm{C}$ unter Rühren zu $4.60 \mathrm{~g}(20.0 \mathrm{mmol}) \mathbf{5 a}$ und $10 \mathrm{ml}$ (ca. $140 \mathrm{mmol}$ ) 15a in $10 \mathrm{ml} \mathrm{HMPT}$ innerhalb $10 \mathrm{~min}$ $27 \mathrm{ml}(20.0 \mathrm{mmol})$ einer $0.74 \mathrm{M}$ Lösung von $\mathrm{KOCMe}_{3}$ in HMPT, rührt $2 \mathrm{~h}$ bei $0^{\circ} \mathrm{C}$, gießt das Gemisch in $20 \mathrm{ml}$ einer ges. Natriumhydrogencarbonat-Lösung, extrahiert dreimal mit jeweils $20 \mathrm{ml}$ Diethylether, wäscht die vereinigten Etherextrakte dreimal mit jeweils $10 \mathrm{ml}$ Wasser und trocknet sie mit Natriumsulfat. Das nach Einengen erhaltene Produkt wird über Kieselgel mit Chloroform/ Hexan (4:1) chromatographiert, Ausb. $0.21 \mathrm{~g} \mathrm{(7 \% )} \mathrm{5,8-Dihydro-5,8-}$ epoxyisochinolin (20), Schmp. des Pikrats $181^{\circ} \mathrm{C}$ (Lit. $\left.{ }^{17 \mathrm{~b})} 178^{\circ} \mathrm{C}\right)$. ${ }^{1} \mathrm{H}$-NMR $\left(\mathrm{CDCl}_{3}\right): \delta=8.44(\mathrm{~s}, 1 \mathrm{H}, 1-\mathrm{H}), 8.28\left(\mathrm{~d}, J_{3,4}=4.6 \mathrm{~Hz}\right.$, $1 \mathrm{H}, 3-\mathrm{H}), 7.21(\mathrm{~d}, 1 \mathrm{H}, 4-\mathrm{H}), 7.00(\mathrm{~d}, 2 \mathrm{H}, 6-7-\mathrm{H}), 5.80$ und $5.73(2 \mathrm{~s}$, $2 \mathrm{H}, 5-, 8-\mathrm{H})$

b) Aus $1.05 \mathrm{~g}(4.56 \mathrm{mmol}) 5 \mathrm{a}$ und $3 \mathrm{ml}$ (ca. $41 \mathrm{mmol}) 15 \mathrm{a}$ in $3 \mathrm{ml}$ HMPT sowie $0.51 \mathrm{~g}$ (4.54 mmol) $\mathrm{KOCMe}_{3}$ in $7 \mathrm{ml} \mathrm{HMPT} \mathrm{wird}$ nach analoger Umsetzung wie unter a) beschrieben der erhaltene Etherextrakt mittels einer GC/MS-Analyse identifiziert. - GC/MS (El, $70 \mathrm{eV}): 4 a(158.0): m / z=157 / 9(20 / 17)\left[\mathrm{M}^{+}\right], 78$ (100), 51 (99); 20 (145.2): $145(10)\left[\mathrm{M}^{+}\right], 117(27), 115$ (100), $90(80), 89(84)$; 4Brompyridin (21) (158.0): 157/9 (22/21) [ $\left.\mathrm{M}^{+}\right], 78(100), 51(89), 151$ (2); 4-Brom-3-(trimethylsilyl)pyridin (22) (230.2): 229/231 (1/2) $\left[\mathrm{M}^{+}\right], 214 / 216(29 / 27), 134$ (65), $107(100), 92$ (21), 73 (95); 3- bzw. 4-tert-Butylpyridin (23 bzw. 24) (151.2): $151(2 / 0.5)\left[\mathrm{M}^{+}\right], 96(83 /$ $100), 95(100 / 48), 57(72 / 65)$.

Umsetzung von 3-Brom-2,4-bis(trimethylsilyl)pyridin (7a) mit

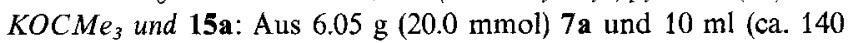
mmol) $15 \mathrm{a}$ in $10 \mathrm{ml} \mathrm{HMPT}$ sowie $27 \mathrm{ml}(20.0 \mathrm{mmol})$ einer $0.74 \mathrm{M}$ Lösung von $\mathrm{KOCMe}_{3}$ in HMPT wie bei der Umsetzung von 5a mit $\mathrm{KOCMe}_{3}$ und 15a beschrieben, jedoch wird anstelle wäßriger Aufarbeitung das Reaktionsgemisch mit $150 \mathrm{ml}$ Hexan verdünnt und vom Niederschlag über eine Vakuumfritte abgesaugt. Die gaschromatographische Untersuchung des Filtrats zeigte quantitativen Umsatz, 5,8-Dihydro-1-(trimethylsilyl)-5,8-epoxyisochinolin (25) als Hauptprodukt und wenig 20 (ca. 10\% bezogen auf 25). Nach Entfernen des Lösungsmittels bei 16 Torr wurde der Rückstand i. Vak. destilliert und anschließend aus Hexan umkristallisiert, Ausb. $1.81 \mathrm{~g}$ (42\%) 25, Sdp. $52-76^{\circ} \mathrm{C} / 10^{-3}$ Torr, Schmp. $67-69^{\circ} \mathrm{C}$ (Hexan). -
${ }^{1} \mathrm{H}-\mathrm{NMR}\left(\mathrm{CDCl}_{3}\right): \delta=8.330\left(\mathrm{~d}, J_{3,4}=4.54 \mathrm{~Hz}, 1 \mathrm{H}, 3-\mathrm{H}\right), 7.065$ $(\mathrm{d}, 1 \mathrm{H}, 4-\mathrm{H}), 6.932$ und $6.875\left(2 \mathrm{dd}, J_{6,7}=5.6, J_{7,8}=1.8 \mathrm{~Hz}, 2 \mathrm{H}\right.$, $6-, 7-\mathrm{H}), 5.835$ und $5.599\left(2 \mathrm{dd}, J_{5,6}=1.6, J_{5,8}=0.7 \mathrm{~Hz}, 2 \mathrm{H}, 5-, 6-\right.$ H).

Umsetzung von 3-Chlor-2,4-bis(trimethylsilyl)pyridin (7b) mit $\mathrm{KOCMe}_{3}$ und 15a: Wie bei der Umsetzung von 5 a mit $\mathrm{KOCMe}_{3}$ und $15 \mathrm{a}$ beschrieben aus $5.28 \mathrm{~g}(20.5 \mathrm{mmol}) 7 \mathrm{~b}$ und $13.9 \mathrm{~g} \mathrm{(204}$ mmol) 15a in $10 \mathrm{ml} \mathrm{HMPT}$ sowie $2.30 \mathrm{~g}(20.5 \mathrm{mmol}) \mathrm{KOCMe}_{3}$, jedoch wurde $\mathrm{KOCMe}_{3}$ portionsweise über eine Feststoffbirne zugegeben. Nach Chromatographie des Rohproduktes über Kieselgel mit Petrolether/Ethylacetat (2:3) Ausb. 1.01 g (35\%) 20, ${ }^{1} \mathrm{H}-\mathrm{NMR}$ spektroskopisch identisch mit dem bei der Umsetzung von $5 \mathbf{a}$ mit $\mathrm{KOCMe}_{3}$ und 15a erhaltenen Produkt.

(5,8-Dihydro-5,8-epoxyisochinolin-1-yl)phenylmethanol (26b): Man gibt bei $0^{\circ} \mathrm{C} \mathrm{zu} 2.08 \mathrm{~g}(8.06 \mathrm{mmol}) 7 \mathrm{~b}$ und $5.48 \mathrm{~g}(80.5 \mathrm{mmol})$ $15 \mathrm{a}$ in $4 \mathrm{ml}$ HMPT über einc Feststoffbirne portionsweise $0.90 \mathrm{~g}$ (8.02 mmol) $\mathrm{KOCMe}_{3}$, rühṛt $2 \mathrm{~h}$ bei $0^{\circ} \mathrm{C}$, gibt dann $0.85 \mathrm{~g}(8.02$ mmol) Benzaldehyd zu und rührt $3 \mathrm{~h}$ bei $100^{\circ} \mathrm{C}$. Das Gemisch wird auf $20 \mathrm{ml}$ einer ges. Natriumhydrogencarbonat-Lösung gegossen, die organische Phase abgetrennt und die wäßrige Phase dreimal mit jeweils $50 \mathrm{ml}$ Diethylether extrahiert. Die vereinigten organischen Phasen werden dreimal mit jeweils ca. $10 \mathrm{ml}$ Wasser gewaschen und mit Natriumsulfat getrocknet. Das Lösungsmittel wird abdestilliert, der Rückstand in 96proz. Ethanol aufgenommen, die Lösung mit 2 Tropfen konz. $\mathrm{HCl}$ versetzt und $3 \mathrm{~h}$ unter Rückfluß erhitzt. Nach Entfernen des Lösungsmittels i. Vak. wird der Rückstand aus Benzol/Hexan (10:1) umkristallisiert, Ausb. $0.81 \mathrm{~g}$ (40\%), Schmp. $163-165^{\circ} \mathrm{C}$.

$$
\begin{array}{ll}
\mathrm{C}_{16} \mathrm{H}_{13} \mathrm{NO}_{2} \text { (251.3) } & \text { Ber. C } 76.48 \text { H } 5.21 \text { N } 5.57 \\
& \text { Gef. C } 76.65 \text { H } 5.20 \text { N } 5.53
\end{array}
$$

Gaschromatographisch werden 2 vollständig getrennte Signale im Verhältnis 1:1 (FID) nachgewiesen, die durch Chromatographie über Kieselgel mit $n$-Hexan/Chloroform (1:1) in jeweils geringe Mengen der reinen Komponenten (diastereomere Enantiomerenpaare) getrennt werden. Beide Produkte werden aus Toluol umkristallisiert, Ausb. 50.3 mg 26 b' (Reinheit 100\%, mittels Kap.-GC bestimmt), Schmp. $169^{\circ} \mathrm{C}$. ${ }^{1} \mathrm{H}-\mathrm{NMR}\left(\mathrm{CDCl}_{3}\right): \delta=8.257$ (d, $\left.J_{3,4}=4.64 \mathrm{~Hz}, 1 \mathrm{H}, 3-\mathrm{H}\right), 7.384-7.263(\mathrm{~m}, 5 \mathrm{H}, \mathrm{Ph}), 7.175(\mathrm{~d}, 1 \mathrm{H}$, $4-\mathrm{H}), 6.781\left(\mathrm{dd}, J_{6,7}=5.52, J_{5,6}=1.93 \mathrm{~Hz}, 1 \mathrm{H}, 6-\mathrm{H}\right), 6.167(\mathrm{dd}$, $\left.J_{7,8}=1.90 \mathrm{~Hz}, 1 \mathrm{H}, 7-\mathrm{H}\right), 5.834(\mathrm{~s}, 1 \mathrm{H}, \mathrm{CH}), 5.656(\mathrm{dd}, 1 \mathrm{H}, 8-\mathrm{H})$, 5.492 (dd, 1 H, 5-H), 5.2 (s, 1 H, OH). - Ausb. 22.7 mg 26b" (Reinheit $97 \%$, mittels Kap.-GC bestimmt), Schmp. $149^{\circ} \mathrm{C} .-{ }^{1} \mathrm{H}-\mathrm{NMR}$ $\left(\mathrm{CDCl}_{3}\right): \delta=8.228\left(\mathrm{~d}, J_{3,4}=4.58 \mathrm{~Hz}, 1 \mathrm{H}, 3-\mathrm{H}\right), 7.389-7.261(\mathrm{~m}$, $5 \mathrm{H}, \mathrm{Ph}), 7.159(\mathrm{~d}, 1 \mathrm{H}, 4-\mathrm{H}), 6.911\left(\mathrm{dd}, J_{6,7}=5.56, J_{7,8}=1.81 \mathrm{~Hz}\right.$, $1 \mathrm{H}, 7-\mathrm{H}), 6.798\left(\mathrm{dd}, J_{5,6}=1.86 \mathrm{~Hz}, 1 \mathrm{H}, 6-\mathrm{H}\right), 5.769(\mathrm{~s}, 1 \mathrm{H}, \mathrm{CH})$, 5.657 (dd, $1 \mathrm{H}, 5-\mathrm{H}), 5.524$ (dd, $1 \mathrm{H}, 8-\mathrm{H}), 4.8(\mathrm{~s}, 1 \mathrm{H}, \mathrm{OH})$.

\section{CAS-Registry-Nummern}

1: 109-04-6 / 2: 84199-54-2 / 3: 134391-66-5/4a: 626-55-1/4b: 626-60-8 / 5a: 134391-67-6/6a: 134391-68-7 / 7a: 134391-69-8/ 7b: 79698-59-2 / 8: 142-08-5 / 9a: 134391-70-1/9b: 134391-76-7/ 10: $109-00-2$ / 11 a: $134391-71-2$ / 11 b: 134391-77-8 / 12: 108-96-3/ 13: 134391-72-3 / 14: 27248-04-0 / 15a: 110-00-9 / 15b: 625-86-5 / 15c: $5471-63-6$ / 17: 17379-48-5 / 18: 65007-00-3 / 19b: 8419956-4 / 20: 40247-40-3 / 21: 1120-87-2/22: 134391-73-4/23: 3803178-6 / 24: 3978-81-2 / 25: 134391-74-5/26b: 134391-75-6/26 b': 134391-79-0 / 26 b': 134391-80-3/ Trimethylsilylchlorid: 75-77-4 ; 2-Brom-4-pyridinylphenylmethanol: 134391-78-9

\footnotetext{
${ }^{1)}$ W. Daub, Dissertation, Univ. Stuttgart, 1985.

2) 2a) R. W. Hoffmann, Dehydrobenzene and Cycloalkynes, Verlag Chemie, Academic Press, Weinheim, New York, London 1967. - ${ }^{2 b)}$ W. Adam, A. Grimison, R. Hoffmann, J. Am. Chem.
} 
Soc. 91 (1969) 2590. - ${ }^{2 c)}$ T. Kauffmann, R. Wirthwein, Angew. Chem. 83 (1971) 21; Angew. Chem. Int. Ed. Engl. 10 (1971) 20. 2d) M. G. Reinecke, Tetrahedron 38 (1982) 427

3) 3a) M. Mallet, G. Quéguiner, Tetrahedron 38 (1982) 3035. - ${ }^{36)} \mathrm{C}$. May, C. J. Moody, Tetrahedron Lett. 26 (1985) 2123.

4) H. H. Nam, G. L. Leroi, J. Am. Chem. Soc. 110 (1988) 4096.

5) B. Jamart-Gregoire, C. Leger, P. Caubere, Tetrahedron Lett. 31 (1990) 7599 .

6) 6a) G. W. J. Fleet, I. Fleming, J. Chem. Soc. C 1969, 1758. ${ }^{5 b)}$ M. J. Pieterse, H. J. den Hertog, Rec. Trav. Chim. Pays-Bas 80 (1961) 1376.

7) 7a) M. J.S. Dewar, W.-K. Li, J. Am. Chem. Soc. 96 (1974) 5569. 7b) T. Yonezawa, H. Konishi, H. Kato, Bull. Chem. Soc. Jpn. 42 (1969) 933. - ${ }^{7 c)}$ M. J. S. Dewar, G. P. Ford, J. Chem. Soc., Chem. Commun. 1977, 539.

${ }^{8)}$ F. Effenberger, W. Daub, Chem. Ber. 124 (1991) 2113, voranstehend.

9) 9a) G. W. Gribble, M. G. Saulnier, Tetrahedron Lett. 21 (1980) 4137. - ${ }^{9 b)}$ F. Marsais, B. Laperdrix, T. Güngör, M. Mallet, G. Quéguiner, J. Chem. Res. (M) 1982, 2863. - ${ }^{9 c}$ F. Marsais, G. Quéguiner, Tetrahedron 39 (1983) 2009.

10) 10a) C. Eaborn, K. L. Jaura, D. R. M. Walton, J. Chem. Soc. 1964 1198. - ${ }^{10 b)}$ D. Häbich, F. Effenberger, Synthesis 1979, 841.
11) O. Kikuchi, H. Togo, N. Furukawa, Bull. Chem. Soc. Jpn. 60 (1987) 3785 .

12) 2al D. S. Noyce, J. A. Virgilio, J. Org. Chem. 37 (1972) 2643. 12b) P. P. Wickham, K. H. Hazen, G. Guo, G. Jones, K. H. Reuter, W. J. Scott, J. Org. Chem. 56 (1991) 2045.

13) Y. Himeshima, T. Sonoda, H. Kobayashi, Chem. Lett. 1983, 1211

14) A. R. Katritzky, J. M. Lagowski, Chemie der Heterocyclen, S. 52, 62, Springer Verlag, Berlin, Heidelberg, New York 1968.

15) L. R. Subramanian, M. Hanack, L. W. K. Chang, M. A. Imhoff, P. v. R. Schleyer, F. Effenberger, W. Kurtz, P. J. Stang, T. E. Dueber, J. Org. Chem. 41 (1976) 4099.

16) F. Effenberger, A. Krebs, P. Willrett, Publikation in Vorbereitung.

17) 17a) M. Mallet, G. Quéguiner, Tetrahedron 35 (1979) 1625. 17b) T. Kauffmann, F.-P. Boettcher, Chem. Ber. 95 (1962) 949. 17c) M. Mallet, F. Marsais, G. Quéguiner, P. Pasteur, C. R. Acad Sci., Ser. C 275 (1972) 1439.

${ }^{18)}$ G. Simchen, J. Pfletschinger, Angew. Chem. 88 (1976) 444; Angew. Chem. Int. Ed. Engl. 15 (1976) 428. 